\title{
Fast Motion Tracking of Tagged MRI Using Angle-Preserving Meshless Registration
}

\author{
Ting Chen ${ }^{1}$, Xiaoxu Wang ${ }^{2}$, Dimitris Metaxas ${ }^{2}$, and Leon Axel ${ }^{1}$ \\ ${ }^{1}$ Radiology Department, New York University Medical Center \\ 650 First Ave, New York City, NY, 10016, USA \\ ${ }^{2}$ Computer Science Department, Rutgers University \\ 617 Bowser Road, Piscataway, NJ, 08854, USA
}

\begin{abstract}
Fast tracking of motion is the key step towards tagged MRIbased quantitative cardiac analysis. Existing motion tracking approaches, including the widely used HARP method, are either time consuming or qualitatively inconsistent, or both. We present in this paper a new fast motion tracking method based on a meshless kernel. For MR image sequences containing multiple image frames, tag intersections are automatically detected in all frames and indexed in the first frame. Then a thin plate spline approach is used to establish a point-to-point correspondence between tag intersections in the initial and the current frame. Lastly, we use a meshless registration kernel to generate a dense displacement map that minimizes the residual of sparse motion at intersections. To further improve the motion tracking, we develop a special technique to preserve tangential angles of tags at tag intersections. We tested our new method on both numerical phantoms and in vivo heart data. The motion tracking results are evaluated against the ground truth and manually drawn tags. Clinical application potential is demonstrated by cardiac strain analysis based on the proposed methodology.
\end{abstract}

Keywords: tagged MRI, motion tracking, meshless, registration.

\section{Introduction}

The diagnosis and treatment of heart diseases can benefit from the quantitative analysis of the myocardial strain, and the strain is derived from the myocardial motion during the cardiac cycle. For this reason, a variety of imaging techniques have been developed to encode the motion within the myocardium in images, so that the in vivo heart motion field can be reconstructed later, using image processing techniques. Tagged MRI (tMRI) 3] 4] has long been viewed as one of the most effective ways to reveal the in vivo heart motion pattern, but currently only limited applications have been developed based on tagged MRI for clinical practice, partly due to the tedious manual intervention required during the traditional process of motion extraction from images.

One currently available motion tracking method, the Harmonic Phase imaging (HARP) 9, extracts the cardiac motion from tagged heart MRI by assessing the local effective phase inside the myocardium caused by the tag pattern. It 
uses the inverse Fourier transform of one harmonic peak of the MR image in the frequency domain to estimate the local tag phase distribution in the spatial domain, and then converts the phase into displacement in order to derive the strain over the cardiac cycle. Two major problems of the HARP approach are: 1) it is vulnerable to large deformation because of its assumption of fixed tag spacing and orientation during the cardiac cycle; and 2) HARP is sensitive to local noises. As a result, besides the previously reported bifurcation in the HARP created phase map, the strain map generated by HARP requires a circumferential smoothing for noise removal [16]: the procedure itself is error prone since it inevitably over smooths the strain distribution.

Other motion tracking approaches have been developed based on either Gabor filter [1] banks and/or deformable models [6]. Both approaches partly improve the quality of the reconstructed displacement. The Gabor methods [13] [14] are similar to HARP. However, to adapt to the local change of tag orientation and spacing, multiple filters with varying parameters are applied to the image in the frequency domain. The local phase, which will be converted into displacement, is determined using only the parameters of filters with the maximum output. The deformable model-based methods [8] 10, 14] track the movement of tags directly. Motions of two sets of tags perpendicular to each other are tracked using spline models and combined to get the $x$ - and $y$-components of the dense displacement in 2D images. These methods do not need the unwrapping process and are usually more accurate than phase-based methods because they converge directly with image data.

However, both methods have their weakness. The Gabor filters' performance falters near the myocardial boundaries since the filter may include irrelevant image information from outside the myocardium. In addition, the Gabor filters' output still needs to be unwrapped before being converted into displacement. This process needs special precaution to prevent potential errors. The deformable model-based method, on the other hand, is driven by local gradients, so that when there is large motion between two frames, the deformable model may 'jump' and mismatch tags and splines.

We present here an alternative method for motion tracking by first sampling the motion at tag intersections, and then solving for the displacement field using the meshless registration. Previous research has been done to address registration and 3D reconstruction problems using the meshless method [7] [12] [15], which is relatively time efficient and more adaptive to the reconstruction of complicate motion fields. In our framework, the motion between frames is first sampled as point-wise motions at tag intersections, using the robust point matching (RPM) method [11. Then, we use the meshless registration [15] to reconstruct a motion field that is: 1) dense, 2) continuous and smooth everywhere inside the myocardium, and 3) minimizing the residual error of motion at tag intersections. To further improve the reconstructed motion, we introduce a new technique that preserves the local motion rotation angle at tag intersections during the registration. 
The paper is organized as follows: section 2 introduces the framework of the new meshless based motion tracking method; section 3 presents experimental results on a numerical phantom and then demonstrates the potential clinical application of the method on tagged MRI analysis; in section 4 we draw our conclusions.

\section{Method}

For simplification, we will introduce the 2D motion tracking although the method can be easily extended into 3D and preliminary study on 3D motion tracking has started. In this paper, we only discuss the motion tracking around the LV and we assume the myocardium around the LV (excluding the papillary muscle) has already been segmented from the background.

\subsection{Tag Intersection Detection}

The motion tracking starts with the detection and indexing of the tag intersections in image frames. We use the Gabor filters as indicated in [14] to generate two sets of phase maps for each frame. Each phase map ranges $[-p i, p i)$, with $-p i$ resembling a mid-line of tags. The tag intersection points are detected as the local minima in the summation of these two phase maps. For the first frame, where there is very little deformation of tags, we can index every tag intersection and store the index in a $2 \mathrm{D}$ array, in which rows and columns resemble horizontal and vertical tags, respectively.

\subsection{Motion Tracking}

We track the myocardial motion in two steps. First we find the correspondence between tag intersections in different image frames using a modified RPM method.

Given two frames, we denote the tag intersections in them as $p$ and $q$, respectively. The RPM is based on the minimization of the least mean square function:

$E(Y, f)=\sum_{i=1}^{M} \sum_{k=1}^{N} y_{i k}\left\|q_{k}-f\left(p_{i}\right)\right\|^{2}+\lambda\|f\|^{2}+T \sum_{i=1}^{M} \sum_{k=1}^{N} y_{j k} \log y_{i k}-\zeta \sum_{i=1}^{M} \sum_{k=1}^{N} y_{i k}$

where $y$ is a fuzzy correlation matrix whose element $y_{i k}=\frac{1}{T} \exp \left(\frac{-\left\|q_{k}-p_{i}^{\prime}\right\|^{2}}{2 T}\right)$ satisfies the constraint $\sum_{i=1}^{M+1} y_{i k}=1$ for $k=1,2, \ldots, N$ and $\sum_{k=1}^{N+1} y_{i k}=$ 1 for $i=1,2, \ldots, M$ with $y_{i k} \in[0,1]$, and $f$ is a point-to-point deformation function. In addition, an entropy term $T \sum_{i=1}^{M} \sum_{k=1}^{N} y_{i k} \log y_{i k}$ in which $T$ is the temperature according to deterministic annealing: it decreases during the point matching so that $y$ shifts from fuzzy to binary array. $\lambda$ and $\zeta$ are both positive weights to keep the final solution smooth and prohibit too many outliers (points 
with no match in the other frame). The value of $\zeta$ changes at points close to the myocardial boundary since the probability that they are outliers is bigger due to through plane motion. We solve this energy function by splitting $f$ into global and local displacement components using the $Q R$ decomposition [5]. The final outcome will be a binary correlation matrix between $p$ and $q$. For the purpose of computing the Lagrangian strain, $p$ are always intersections in the first frame.

Ignoring all the outliers in $p$ and $q$, we start the generation of the displacement map by minimizing the following energy:

$$
E=\sum_{j} \sum_{i=1}^{N} w_{i j}\left\|f_{j}\left(p_{i}\right)-q_{i}\right\|^{2}
$$

where $j$ is a pixel within the myocardium in the first frame, and $i$ is the common index of tag intersections in both frames. $f_{j}$ is the affine transformation at pixel $j$, and $w$ is an array of weights. We enforce $p_{i}$ and $q_{i}$ to be row vectors so that $p_{i}=\left(p_{i x}, p_{i y}\right)$. To accommodate potential anisotropic situations, we define

$$
w_{i j}=\exp -\left(\left(\frac{p_{i x}-j_{x}}{\sigma_{x}}\right)^{2}+\left(\frac{p_{i y}-j_{y}}{\sigma_{y}}\right)^{2}\right)
$$

Empirically we set $\sigma_{x}$ and $\sigma_{y}$ equal to the initial tag spacing. Assume that the motion at each pixel is an affine transformation and can be written as:

$$
f_{j}(a)=a M+T
$$

where $a$ is the coordinate vector. We can solve for $f_{j}$ by minimizing all the terms in EqL that contain $j$ :

$$
E_{j}=\sum_{i} w_{i j}\left\|f_{j}\left(p_{i}\right)-q_{i}\right\|^{2}
$$

Recalling the relative location information in the index array, we can estimate the derivatives of displacement at tag intersections using the variation of the displacement between its neighbor intersections. For example, for the intersection of the $m$ th horizontal tag and $n$th vertical tag, defining the displacement in the $x$ - and $y$ - directions as $u$ and $v$, we have:

$$
\left.\frac{\partial u}{\partial x}\right|_{q(m, n)}=\frac{q(m, n+1)-q(m, n-1)}{2 * \sigma_{x}}
$$

We can similarly find $\frac{\partial u}{\partial y}, \frac{\partial v}{\partial x}$, and $\frac{\partial v}{\partial y}$ at tag intersections. To integrate the effect of the derivative information, we replace $q$ in Eq 5 with $\hat{q}$ :

$$
\hat{q}_{i}=q_{i}+\left(j_{x}-x_{i}, j_{y}-y_{i}\right) \partial q
$$

where $\partial q=\left|\begin{array}{l}\frac{\partial u}{\partial x} \frac{\partial v}{\partial x} \\ \frac{\partial u}{\partial y} \frac{\partial v}{\partial y}\end{array}\right|$. 
We solve for $f_{j}$ by minimizing the updated Eq5. The minimum is achieved when derivatives with regard to free variables as shown in Eq, 4 equal to zero. First we minimize w.r.t the translation $T$ :

$$
T=\hat{q}_{*}-p_{*} M
$$

where $\hat{q}_{*}$ and $p_{*}$ are weighted centroids, such that $\left.\hat{q}_{*}\right|_{j}=\frac{\sum_{i} w_{i j} \hat{q}_{i}}{\sum_{i} w_{i j}}$ and $\left.p_{*}\right|_{j}=$ $\frac{\sum_{i} w_{i j} p_{i}}{\sum_{i} w_{i j}}$. We then substitute $T$ in Eq[5 and rewrite $f_{j}=\left(\left[j_{x}, j_{y}\right]-p_{*}\right) M+\hat{q}_{*}$. Now we can rewrite Eq[5] as:

$$
E_{j}=\sum_{i} w_{i j}\left\|\tilde{p}_{i} M-\tilde{q}_{i}\right\|^{2}
$$

where $\tilde{p}_{i}=p_{i}-p_{*}$ and $\tilde{q}_{i}=\hat{q}_{i}-\hat{q}_{*}$. It is relatively straightforward to find the solution of $M$ using the derivative of $E_{j}$ w.r.t $M$. We give the solution to $M$ and $f_{j}$ here:

$$
\begin{gathered}
M=\left(\sum_{i}{\tilde{p_{i}}}^{T} w_{i j}{\tilde{p_{i}}}^{-1} \sum_{k} w_{k j}{\tilde{p_{k}}}^{T} \tilde{q_{k}}\right. \\
f_{j}(a)=\sum_{k} A_{k} \tilde{q_{k}}+\hat{q}_{*}
\end{gathered}
$$

where $A_{k}=\left(a-p_{*}\right)\left(\sum_{i}{\tilde{p_{i}}}^{T} w_{i j} \tilde{p}_{i}\right)^{-1} w_{k j}{\tilde{p_{k}}}^{T}$. It is clear that the computation of $A_{k}$ only depends on $w$ and $p$, both of which are known at the start of the motion tracking so that we need to calculate $A_{k}$ just once for tracking of the whole cardiac cycle, which typically consists of around 20 frames.

The pseudo code of the method is:

Initialize. Extract tag intersections $t_{1}$ in the initial (undeformed) frame of the image sequence

WHILE current frame $i$ not at the end of the image sequence Extract tag intersections $p_{i}$ in the next frame using $\mathbf{G a b o r}(\mathbf{i})$

Use $\mathbf{R P M}\left(\mathbf{p}_{\mathbf{1}}, \mathbf{p}_{\mathbf{i}}\right)$ to build the sparse displacement $C_{i}$ at frame $i$ Use $\mathbf{D M}\left(\mathbf{i}, \mathbf{C}_{\mathbf{i}}\right)$ to reconstruct the dense displacement field ENDWHILE

\subsection{Angle-Preserving Motion Tracking}

We extend the method by incorporating the tangents of the tags at intersections. Consider one tag intersection $p_{i}=\left(p_{x}, p_{y}\right)$ and its deformed correspondence $q_{i}=\left(q_{x}, q_{y}\right)$. When we move $p_{i}$ a short distance in the $x$ - direction to $p_{i}^{\prime}=$ $\left(p_{x}+\Delta x, p_{y}\right)$, we can compute the location of the new location of $q_{i}$ using Eq4 Because $\Delta x$ is small, we can assume $M$ and $T$ remain constant for $p_{i}$ and $p_{i}^{\prime}$. The new location of $q_{i}^{\prime}=p_{i}^{\prime} M+T=q_{i}+\Delta x\left(M_{11}, M_{12}\right)$, where $M_{i j}$ are elements of $M$. It is clear that to preserve the tangent of the horizontal tag at $q_{i}$, we need $\frac{M_{12}}{M_{11}}=\frac{\partial v}{\partial x}$. Similarly we can get $\frac{M_{21}}{M_{22}}=\frac{\partial u}{\partial y}$ for the vertical tag. It is easy for us 
to compute the tangents of the tags (in this paper we perform tag fitting around intersections using the image gradient, as described in [14]).

Let $\frac{\partial v}{\partial x}=c_{1}$ and $\frac{\partial u}{\partial y}=c_{2}$. We have

$$
M^{T}=\left|\begin{array}{l}
M_{11} M_{21} \\
M_{12} M_{22}
\end{array}\right|=\left|\begin{array}{cc}
1 & c_{1} \\
c_{2} & 1
\end{array}\right| \begin{array}{ll}
\alpha & 0 \\
0 & \beta
\end{array} \mid .
$$

The first array on the right hand side of the equation is known at each tag intersection, but $\alpha$ and $\beta$ are unknowns to us. Therefore we can preserve the tag angles at intersections by converting the search of $M$ into solving $\alpha$ and $\beta$. The solving processes of $\alpha$ and $\beta$ are similar to $M$. Define

$$
\hat{p}_{i}=p_{i}\left|\begin{array}{cc}
1 & c_{1} \\
c_{2} & 1
\end{array}\right|
$$

update $\tilde{p}_{i}=\hat{p}_{i}-\hat{p}_{*}$ and use Eq[10 with the new $\tilde{p}_{i}$, we have

$$
\begin{aligned}
& \alpha=\left(\sum_{i} \tilde{p}_{i x}^{2} \cdot w_{i j}\right)^{-1} \sum_{k} w_{k j} \tilde{p}_{k x} \tilde{q}_{k x} \\
& \beta=\left(\sum_{i} \tilde{p}_{i y}^{2} \cdot w_{i j}\right)^{-1} \sum_{k} w_{k j} \tilde{p}_{k y} \tilde{q}_{k y}
\end{aligned}
$$

By solving $\alpha$ we can generate the $x$ - component in the displacement (or $u$ ), and by solving $\beta$ we get the $y-$ component $(v)$.

\section{Experimental Results and Discussion}

We tested the new motion tracking method on numerical phantoms as well as in vivo images. The numerical phantom has the shape of an annulus. Horizontal and vertical darker stripes have been generated inside the phantom as tags, and deform based on a pre-computed displacement field in a sequence of frames simulating the cardiac motion. We used our new method to track the motion in the phantom and compared the result against other motion tracking methods

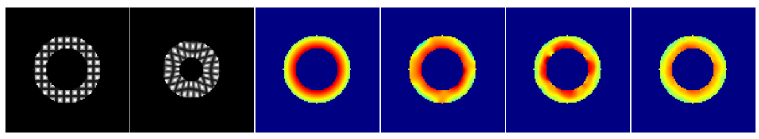

Fig. 1. From left to right: The undeformed phantom; the deformed phantom; true displacement magnitude; displacement generated using the meshless tracking; displacement generated using HARP; and displacement generated using the Gabor method. All displacement images are shown using the same color scale.

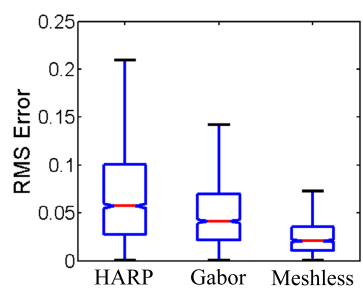

Fig. 2. RMS error of strain in the numerical phantom 
Table 1. The quality comparison of the new motion tracking method against HARP, Gabor based method, and the ground truth. The 2nd row is the true mean displacement magnitude in the phantom at 10 time frames. The 3rd and 4th row are the RMS error in displacement magnitude with the meshless method and its ratio w.r.t the ground truth, respectively. 5th and 6th row: HARP results; and 7th and 8th row: Gabor results.

\begin{tabular}{|c|c|c|c|c|c|c|c|c|c|c|}
\hline Frame & 1 & 2 & 3 & 4 & 5 & 6 & 7 & 8 & 9 & 10 \\
\hline Mean Disp. & 0.0 & 1.1234 & 2.4574 & 4.0282 & 5.0850 & 5.6400 & 5.0902 & 4.0527 & 2.3180 & 0.9968 \\
\hline RMS error & 0.0 & 0.1491 & 0.1936 & 0.1921 & 0.2558 & 0.3570 & 0.3677 & 0.3505 & 0.2578 & 0.2029 \\
\hline Ratio (\%) & NA & 13.27 & 7.88 & 4.77 & 5.03 & 6.33 & 7.22 & 8.65 & 11.12 & 20.36 \\
\hline HARP RMS error & 0.0 & 0.1054 & 0.1655 & 0.2996 & 0.4899 & 0.7120 & 0.6171 & 0.5071 & 0.6309 & 0.8222 \\
\hline HARP Ratio (\%) & NA & 9.38 & 6.74 & 7.44 & 9.63 & 12.62 & 12.12 & 12.51 & 27.22 & 82.48 \\
\hline Gabor RMS error & 0.0 & 0.0954 & 0.1978 & 0.3536 & 0.5644 & 0.7192 & 0.6091 & 0.4104 & 0.2402 & 0.3319 \\
\hline Gabor Ratio (\%) & NA & 8.49 & 8.05 & 8.78 & 11.10 & 12.75 & 11.97 & 10.13 & 10.36 & 33.29 \\
\hline
\end{tabular}

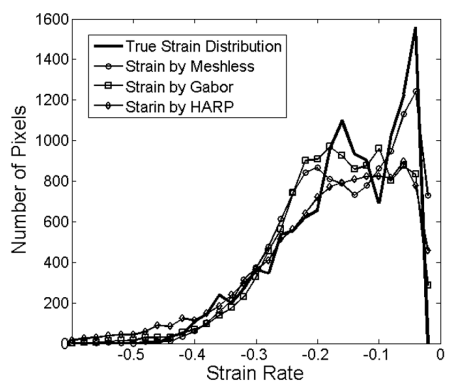

Fig. 3. Strain rate distribution of the numerical phantom
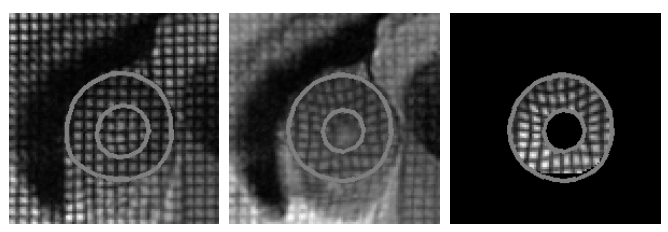

Fig. 4. Left: tMRI at the end of diastole (ED); middle: tMRI at the end of systole (ES); and right: reconstructed ES image based on the motion tracking between the ED and ES frames. There are missing pixels in the regenerated image due to through-plane motion.

including the HARP and the ground truth for evaluation, as shown in Fig 1 and Table1 In addition, we calculated the circumferential strain in the phantom, based on the generated motion, and compared the outcome with other methods and the ground truth. RMS error of the strain and pixel numbers at different strain level are shown in Fig 2 and Fig 3 , respectively, for the new method and two other methods including the HARP. The new motion tracking method outperformed other methods and was very close to the ground truth.

We tested the method on more than 15 tagged MRI data sets from patients and normal volunteers. In Fig 4 we show an image generated using the motion tracking. The new method was also validated by comparing the generated motion with tag lines manually drawn by cardiac experts, and results showed very promising agreement: RMS error between corresponding tag points within the myocardium was under 0.1 pixel.

The method is extremely fast in $2 \mathrm{D}$, it takes less than 35 seconds to track the motion in an image sequence consisting of 10 frames of the size 100 by 100 pixels. For 3D, the timing performance slows down but is still acceptable. Typically for a $3 \mathrm{D}$ data set consisting of $5 \mathrm{SA}$ slices and $3 \mathrm{LA}$ slices, we can reconstruct the 
motion between two frames within two minutes. All experiments are done on a $\mathrm{PC}$ with core 2 duo $\mathrm{CPU}$ at $2.66 \mathrm{GHz}$ main frequency.

\section{Conclusions}

We have developed a new motion tracking method based on meshless registration techniques. The motion between image frames is reconstructed by minimizing the moving least square energy. Tag intersections' initial locations and tags' orientation at intersections are incorporated in order to improve the quality of motion tracking. More validation and algorithm optimization will be performed in 3D in the near future. The new method may have potential for clinical applications, as demonstrated by its performance on real heart data.

\section{References}

1. Gabor, D.: Theory of communication. J. IEE 93(3), 429-457 (1946)

2. Lancaster, P., Salkauskas, K.: Surfaces generated by moving least squares methods. Mathematics of Computation, 141-158 (1981)

3. Axel, L., Dougherty, L.: MR imaging of motion with spatial modulation of magnetization. Radiology 171, 841-845 (1989)

4. Axel, L., Dougherty, L.: Improved method of spatial modulation of magnetization (SPAMM) for MRI of heart wall motion. Radiology 172, 349-350 (1989)

5. Wahba, G.: Spline models for observational data. SIAM, Philadelphia (1990)

6. Metaxas, D.: Physics-based Deformable Models: Application to Computer Vision, Graphics and Medical Imaging. Springer, Heidelberg (1996)

7. Belytschko, T., Krongauz, Y., Organ, D., Fleming, M., Krysl, P.: Meshless methods: An Overview and Recent Developments. Computer Methods in Applied Mechanics and Engineering, 3-47 (1996)

8. Young, A.: Model tags: direct 3D tracking of heart wall motion from tagged magnetic resonance images. Medical Image Analysis, 361-372 (1999)

9. Osman, N.F., McVeigh, E.R., Prince, J.L.: Imaging heart motion using Harmonic Phase MRI. IEEE Trans. on Medical Imaging 19(3), 186-202 (2000)

10. Amini, A.A., Chen, Y., Elayyadi, M., Radeva, P.: Tag surface reconstruction and tracking of myocardial beads from SPAMM-MRI with parametric B-spline surfaces. IEEE Trans. on Medical Imaging 20(2), 94-103 (2001)

11. Chui, H., Rangarajan, A.: A new point matching algorithm for non-rigid registration. Computer Vision and Image Understanding 89(2-3), 114-141 (2003)

12. Müller, M., Keiser, R., Nealen, A., Pauly, M., Gross, M., Alexa, M.: Point based animation of elastic, plastic and melting objects. In: Proceedings of the 2004 ACM SIGGRAPH/Eurographics symposium on Computer animation, pp. 141-151 (2004)

13. Axel, L., Chen, T., Manglik, T.: Dense myocardium deformation estimation for 2D tagged MRI. In: Proceedings of FIMH, pp. 446-456 (2005)

14. Chen, T., Axel, L.: Using Gabor filters bank and temporal-spatial constraints to compute 3D myocardium strain. In: Proceedings of EMBC (2006)

15. Schaefer, S., McPhail, T., Warren, J.: Image deformation using moving least squares. In: Proceedings of the 2006 ACM SIGGRAPH pages, pp. 533-540 (2006)

16. Abd-Elmoniem, K.Z., Parthasarathy, V., Prince, J.L.: Improving HARP cardiac strain mapping using nonlinear diffusion. In: Proceedings of ISMRM (2006) 